\title{
Are psychosocial stressors associated with the relationship of alcohol consumption and all-cause mortality?
}

Esther Ruf ${ }^{1}$, Jens Baumert ${ }^{1}$, Christa Meisinger ${ }^{1,2}$, Angela Döring ${ }^{3}$, Karl-Heinz Ladwig ${ }^{1,4^{*}}$ and for the MONICA/KORA investigators

\begin{abstract}
Background: Several studies have shown a protective association of moderate alcohol intake with mortality. However, it remains unclear whether this relationship could be due to misclassification confounding. As psychosocial stressors are among those factors that have not been sufficiently controlled for, we assessed whether they may confound the relationship between alcohol consumption and all-cause mortality.

Methods: Three cross-sectional MONICA surveys (conducted 1984-1995) including 11,282 subjects aged 25-74 years were followed up within the framework of KORA (Cooperative Health Research in the Region of Augsburg), a population-based cohort, until 2002. The prevalences of diseases as well as of lifestyle, clinical and psychosocial variables were compared in different alcohol consumption categories. To assess all-cause mortality risks, hazard ratios (HRs) were estimated by Cox proportional hazards models which included lifestyle, clinical and psychosocial variables.

Results: Diseases were more prevalent among non-drinkers than among drinkers: Moreover, non-drinkers showed a higher percentage of an unfavourable lifestyle and were more affected with psychosocial stressors at baseline. Multivariable-adjusted HRs for moderate alcohol consumption versus no consumption were 0.74 (95\% confidence interval (Cl): 0.58-0.94) in men and 0.87 (95\% Cl: 0.66-1.16) in women. In men, moderate drinkers had a significantly lower all-cause mortality risk than non-drinkers or heavy drinkers $(p=0.002)$ even after multivariable adjustment. In women, moderate alcohol consumption was not associated with lowered risk of death from all causes.

Conclusions: The present study confirmed the impact of sick quitters on mortality risk, but failed to show that the association between alcohol consumption and mortality is confounded by psychosocial stressors.
\end{abstract}

Keywords: Alcohol, Confounding, Mortality, Psychosocial stressors

\section{Background}

A repeated observation in diverse populations is that light to moderate alcohol consumption provides a window of protection in which adverse health effects are outweighed by benefits. Numerous epidemiological studies reported J- or U-shaped curves when describing the

\footnotetext{
* Correspondence: ladwig@helmholtz-muenchen.de

IInstitute of Epidemiology II, Helmholtz Zentrum München, German Research Center for Environmental Health, Ingolstädter Landstr. 1, 85764 Neuherberg, Germany

${ }^{4}$ Department of Psychosomatic Medicine and Psychotherapy, Klinikum rechts der Isar, Technische Universität München, Langerstr. 3, 81675 München, Germany

Full list of author information is available at the end of the article
}

association between levels of alcohol consumption and allcause or cardiovascular mortality [1-4], showing an increased mortality risk among abstainers and heavy drinkers compared to light or moderate drinkers. These risk patterns have been shown to be less pronounced in women than in men [5].

Several pathophysiological mechanisms are considered to be responsible for the beneficial effect of moderate alcohol consumption, which increases serum HDL and lowers fibrinogen and tryglyceride concentrations as well as blood pressure [6]. In addition to the protective effect of moderate drinking derived from pathophysiological features of alcohol [7] and the inclusion of subjects who

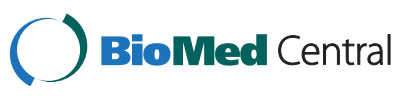


abstain because of illness (so-called 'sick quitters') in the non-drinking group [8], unmeasured confounders might be responsible for this alcohol-mortality relationship. Although there is evidence that shows beneficial trends in biomarker concentrations as a result of low to moderate alcohol intake [9], psychosocial stressors are among those factors that have not been sufficiently controlled for, indicating that the potential confounding of the relationship between alcohol and all-cause mortality by these stressors has not yet been clearly assessed.

Andreasson [10] suspects that J-shaped curves may be the result of complex associations between psychosocial stressors, other potential confounding factors and health conditions. There is broad evidence that psychosocial stressors are associated with increased cardiovascular and overall mortality, as shown for depression [11-13], work stress $[14,15]$, impaired self-perceived health $[16,17]$ and, less consistently, for anxiety $[18,19]$. Several cross-sectional studies have indicated that psychosocial variables have the same J- or U-shaped curves in relation to alcohol consumption as to mortality benefits [20-26]: non-drinkers and heavy drinkers alike may experience higher levels of depression and anxiety, suffer more frequent from bodily pain and perceive themselves to be less healthy, to have less vitality, and to be generally less sociable than moderate drinkers. Hence, the established J- or U-shaped relationship between alcohol consumption and mortality might be confounded by psychosocial stressors.

Daily living may exert numerous psychosocial stressors on the individual, including external stressors (e.g. work stress, low employment status, living alone), and internal stressors (e.g. depressive symptoms, intrusive impact of bodily symptoms). Minimizing emotional response to stress may lower psychological distress [23]. Higher levels of psychological distress are associated with the frequency and the volume of alcohol consumption [27]. Therefore, moderate alcohol consumption as a sign of a person's favourable lifestyle may serve as a surrogate marker for a spectrum of positive mental health factors that attenuates the effect of chronic stressors on health [18]. This general positive health behaviour and the reduced exposure of psychosocial stressors may be most important as this is especially likely to reduce risk of illness.

While lifestyle and clinical risk factors have been considered in many of the studies investigating the association of alcohol consumption and mortality, there is a substantial lack of studies that controlled for psychosocial stressors. Therefore, the present study aims to set its focus to this class of potential confounders. Using data from the population-based MONICA/KORA Augsburg Cohort Study, we investigated if psychosocial stressors confound the relationship between alcohol consumption and all-cause mortality by comparing models with and without psychosocial stressors included.

\section{Methods}

\section{Study design}

The data of the present study were derived from the population-based MONICA (MONItoring trends and determinants in CArdiovascular disease) Augsburg Study as part of the multinational WHO MONICA project [28]. Altogether 13,427 subjects (6,725 men, 6,702 women, response 77\%) aged 25-64 years (S1) and 25-74 years (S2, S3), respectively, randomly drawn from the general population with German nationality of the city of Augsburg and two adjunct counties (Southern Germany), participated in at least one of the three independent crosssectional surveys, conducted in 1984/85 (S1), 1989/90 (S2) and 1994/95 (S3). All subjects were prospectively followed up within the framework of the Cooperative Health Research in the Region of Augsburg (KORA) until 2002 [29]. Mortality was ascertained by regularly checking the vital status of all sampled persons of the MONICA surveys through the population registries. In 1997-1998 and 2002-2003 the health status of all living persons was assessed using follow-up questionnaires. The MONICA surveys S1, S2 and S3 with the baseline examination were approved by the data protection commission following the rules at the time of the examinations $(1984 / 85,1989 / 90$ and 1994/95). The follow-up examinations within the KORA framework were approved by the ethics committee of the Bavarian Medical Association. All studies were performed in accordance with the Declaration of Helsinki. All participants provided written informed consent.

\section{Study population}

Among the MONICA/KORA sample of 13,427 subjects, 12,887 subjects $(96.0 \%)$ had available psychosocial data. Of those, 3,917 (30.4\%) were from S1, 4,539 (35.2\%) from S2 and 4,431 (34.4\%) from S3. The psychosocial data set extended the MONICA core design and followed recommendations given by the MONICA steering committee [30]. Eight participants had missing data in alcohol consumption and were excluded to assess the prevalence of diseases, leading to a total of 12,879 subjects.

For mortality analyses, participants suffering from disease conditions or undergoing treatments that require abstinence from alcohol (diabetes $\mathrm{N}=508$, heart failure $\mathrm{N}=628$ ), from severe diseases (myocardial infarction $\mathrm{N}=255$, cancer $\mathrm{N}=142$, only $\mathrm{S} 1$ ) or from diseases indicating possible former high alcohol consumption (liver disease $\mathrm{N}=394$ ) were excluded to avoid bias due to socalled 'sick quitters'. A drop-out analysis revealed that disease burden was highest in the abstainer group, who had higher prevalences of a history of myocardial infarction, heart failure, diabetes and liver disease. No substantial differences with respect to drinking patterns were observed for participants with a history of cancer. A total of 1,599 participants (911 men and 688 women) suffering 
from at least one severe disease condition (which may influence drinking behaviour) were excluded leading to a study population of 11,282 participants (5,540 men and 5,742 women) for mortality analyses. For several variables, analyses were not possible within the whole study group because of missing data or because variables had not been investigated in all three surveys.

\section{Study endpoint}

The study endpoint was all-cause mortality. Death certificates were obtained from the local health departments and were coded for the underlying cause of death using the ninth revision of the International Classification of Diseases (ICD 9) [31]. The duration of the follow-up was calculated as the interval between the baseline examination and death from all-causes, or the date of the last available follow-up information which was drawn from KORA follow-up examinations in 1997-1998 or 2002-2003 or from population registries. The last possible observation date was December 31, 2002. The cohort in the present analysis was followed for an average of 12.0 years (standard deviation 4.4) ranging from 0.1 to 18.2 years. During this observation period, 970 (15.0\%) men and 479 (7.5\%) women had died.

\section{Risk factor assessment at baseline}

Baseline information on sociodemographic variables, alcohol consumption, smoking status, physical activity level, medical history and medication use was assessed by trained medical staff during a standardized interview. Additionally, all participants underwent an extensive standardized medical examination that included the collection of a non-fasting blood sample.

Psychosocial variables were answered by a selfadministered questionnaire.

\section{Alcohol consumption}

In the standardized interview, alcohol intake was assessed by the following questions: 'How much beer/wine/spirits did you drink over the previous weekend (Saturday and Sunday)?', 'How much beer/wine/spirits did you drink on the previous workday (or on the previous Thursday, if Friday was the previous workday)?' Total intake was calculated by multiplying weekday consumption by five and adding this to weekend consumption, applying the following conversions: 1 liter beer $=40 \mathrm{~g}$ alcohol, 1 liter wine = $100 \mathrm{~g}$ alcohol, 1 shot distilled spirits $(0.02$ liter $)=6.2 \mathrm{~g}$ alcohol. Finally, the average number of grams of alcohol consumed per day (g/day) was derived. This 7-day recall method was validated against a 7-day diet record method in a subsample and revealed sufficient validity [1].

For the present analysis, alcohol consumption was classified into three categories: no alcohol consumption (0 g/day), moderate alcohol consumption (0.1-39.9 for men and 0.1-19.9 for women) and heavy alcohol consumption ( $\geq 40 \mathrm{~g} /$ day for men and $\geq 20 \mathrm{~g} /$ day for women) following previous studies regarding cardiovascular and allcause mortality [1,32]. A broader classification into six categories was used when analysing crude death rates (see Figure 1), but was not used for the main analyses due to low case numbers and rather similar mortality risks in the three high alcohol consumption categories (40-59.9, 60.0-79.9, $\geq 80$ g/day).

\section{Lifestyle and clinical variables}

Participants were classified as regular smokers when they reported that they currently smoke at least one cigarette per day.

To assess physical inactivity, participants were classified as 'physically inactive' during leisure time if they did not regularly participate in sports and if they were not active for at least $1 \mathrm{~h}$ per week in summer and winter.

Body height and body weight were determined by trained medical staff following a standardized protocol. Body mass index was calculated as weight in kilograms divided by high in square meters. According to the recommendations of the WHO, obesity was defined as BMI $\geq 30 \mathrm{~kg} / \mathrm{m}^{2}$.

Blood pressure was measured using the right arm in a sitting position using a Hawksley random-zero sphygmomanometer adhering to the WHO MONICA protocol [29]. Actual hypertension was defined as blood pressure value $\geq 140 / 90 \mathrm{mmHg}$ or use of antihypertensive medication, which indicates that the subject was aware of being hypertensive.

A non-fasting venous blood sample was collected from all participants while sitting. Total serum cholesterol (mg/dl) and high-density lipoprotein cholesterol (HDL-C) after precipitation with phosphotungstic acid/Mg2+, were measured by enzymatic methods (CHOD-PAP, Boehringer Mannheim, Germany). Dyslipidemia was defined as a ratio of total cholesterol to HDL-C $\geq 5$.

Finally, history of diabetes, heart failure, myocardial infarction, cancer and liver diseases were assessed by self-

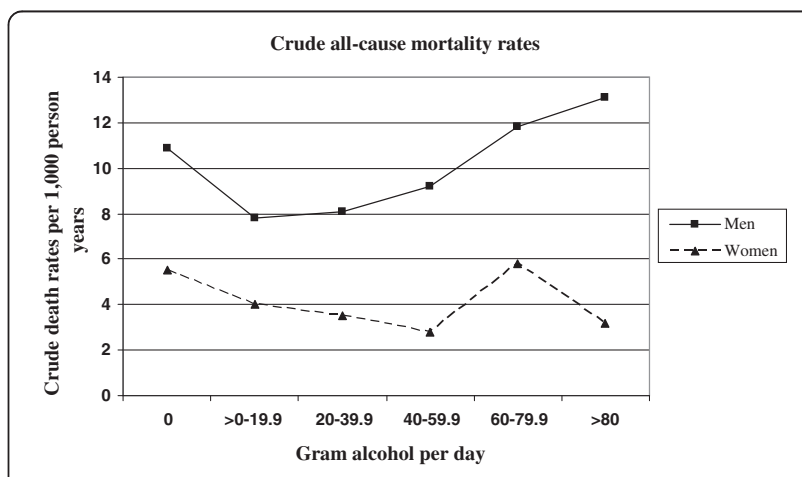

Figure 1 Crude all-cause mortality rates for alcohol consumption in men and women ( $n=11,282$ after exclusion of ill persons at baseline). 
reports of the participants and used for the definition and exclusion of so-called 'sick quitters'.

\section{Psychosocial stressors}

Educational level was categorized into 'low' ( $\leq 12$ years of schooling) and 'high' (> 12 years of schooling). Occupational status was classified into three categories: 'currently employed', 'unemployed' (i.e. subjects seeking work) and 'not employed' (i.e. homemakers or subjects in periods of education, parental leave or retirement). Social support was assessed according the Social Network Index (SNI) initially designed for the Alameda county study [33] comprising marital status, contact with friends and relatives, and an index of close contacts and activities in groups. The combination of all components allows a comprehensive rating from 1 (low SNI) to 4 (high SNI). SNI was classified into three categories using tertiles of the distribution (low, medium, high). Living alone was defined by marital status (living alone or being separated, divorced or widowed). Job strain symptoms, according to Karasek [34], were also classified into three categories using tertiles of the distribution (low, medium, high). Low educational level, unemployment, low social network, living alone, and high job strain were considered as external stressors.

Self-perceived health was directly assessed by the interview question: 'How would you assess your current health condition - excellent, good, fair, poor?' These four categories were dichotomized to an 'excellent/good'- and a 'fair/ poor'-group [35]. Depressive symptoms were assessed using a subscale from the von Zerssen affective symptom check list [36,37]. Subjects in the upper third of the depressive symptom distribution were considered as the index group for subjects with a depressed mood. Severe somatic complaints were examined by nine items from the von Zerssen affective symptom check list [36]. The subscale combined nine items (e.g. sweating, palpitation, numbness, vertigo) ranging from 0 to 3 , leading to a score range of $0-27$. The internal consistency (measured by Cronbach's $\alpha$ ) of the somatic complaints subscale was high. The variable 'level of somatic complaints' was defined by using sex-specific tertiles of the distribution of the somatic complaints subscale (low, medium, high). Low self-perceived health, depressed mood and high level of somatic complaints were chosen as markers of internal stressors.

\section{Statistical analysis}

Since the risk which is attributable to alcohol consumption differs by sex, all analyses were performed separately for men and women.

Crude incidence rates of all-cause mortality were estimated by the person-years method [38]. Cox proportional hazards models were calculated to assess the relative risk of all-cause mortality for moderate and high alcohol consumption compared to no alcohol consumption. The proportional hazards assumption was assessed by plotting the $[-\log ($ survival) $]$ curves for each risk factor showing that proportional hazards could be assumed for all risk factors. Relative risks were computed as hazard ratios (HRs) with 95 percent confidence intervals ( $95 \% \mathrm{CI}$ ). The first 'crude' model included age (continuous) and survey (S1, S2 or S3), followed by the second 'lifestyle/clinical' model which included additionally the lifestyle variables regular smoking (yes or no), physical inactivity (yes or no), and obesity (yes or no) as well as the clinical variables actual hypertension (yes or no) and dyslipidemia (yes or no). The third 'psychosocial stressors' model included age, survey and additionally the psychosocial stressors low educational level ( $\leq$ or $>12$ years), not employed (yes or no), low social network (yes or no), living alone (yes or no), high job strain (yes or no), low self- perceived health (yes or no), depressed mood (yes or no) and high level of somatic complaints (yes or no). The fourth 'full' model included age, survey and all lifestyle, clinical and psychosocial factors. Moreover, we tested possible interactions between alcohol consumption and all psychosocial factors on allcause mortality risk.

Significance tests were 2-tailed. For all statistical analyses a $\mathrm{P}$ value less than 0.05 was considered to be statistically significant. The Akaike's Information Criterion (AIC) was used to assess the goodness of fit of the models. The evaluations were performed with the statistical software package SAS (Version 8.02, SAS-Institute Inc., Cary, NC, USA).

\section{Results}

\section{Descriptive analyses}

In the study population $(\mathrm{N}=11,282)$, a total of 850 (15.3\%) males and 2,401 (41.8\%) females reported no alcohol consumption. A total of 2,833 (51.1\%) men and 2,230 (38.8\%) women were classified to be moderate drinkers. Heavy alcohol consumption was observed in 1,857 (33.5\%) men and 1,111 (19.3\%) women.

Baseline characteristics stratified by alcohol consumption groups from the apparently healthy study sample $(\mathrm{N}=11,282)$, adjusted for age and survey, are displayed in Table 1. The moderate consumption group reported significantly lower percentages in a number of lifestyle and clinical factors (e.g. physical inactivity, obesity, dyslipidemia) in comparison to heavy drinkers and non-drinkers. When considering psychosocial stressors, moderate drinkers reported significantly lower percentages of external stressors such as low educational level, low social network and living alone. Regarding internal stressors, no differences in depressive mood frequency was observed between the alcohol consumption groups in men. In women, moderate drinkers reported significantly less somatic complaints. 
Table 1 Prevalence of medical and lifestyle variables and psychosocial stressors by alcohol consumption in men and women, adjusted for age and survey ( $n=11,282$ after exclusion of diseased participants at baseline)

\begin{tabular}{|c|c|c|c|c|c|c|c|c|}
\hline & \multicolumn{8}{|c|}{ Alcohol consumption (g/day) } \\
\hline & \multicolumn{4}{|c|}{$\begin{array}{c}\text { Men } \\
(n=5,540)\end{array}$} & \multicolumn{4}{|c|}{$\begin{array}{c}\text { Women } \\
(n=5,742)\end{array}$} \\
\hline & $\begin{array}{c}0 \\
(n=850)\end{array}$ & $\begin{array}{l}>0-39.9 \\
(n=2,833)\end{array}$ & $\begin{array}{c}\geq 40 \\
(n=1,857)\end{array}$ & $p$-value* & $\begin{array}{c}0 \\
(n=2,401)\end{array}$ & $\begin{array}{l}>0-19.9 \\
(n=2,230)\end{array}$ & $\begin{aligned} & \geq 20 \\
(n & =1,111)\end{aligned}$ & $p$-value* \\
\hline Regular smoking [\%] & 34.4 & 28.0 & 40.7 & $<0.0001$ & 20.0 & 22.2 & 26.8 & $<0.0001$ \\
\hline Physically inactive [\%] & 56.6 & 51.5 & 56.1 & 0.0015 & 65.5 & 57.6 & 54.7 & $<0.0001$ \\
\hline Obesity [\%] & 19.6 & 16.8 & 16.8 & 0.1222 & 23.6 & 16.2 & 12.1 & $<0.0001$ \\
\hline Actual hypertension [\%] & 39.9 & 39.3 & 46.5 & $<0.0001$ & 30.0 & 27.5 & 29.9 & $<0.0838$ \\
\hline Dyslipidemia [\%] & 52.9 & 44.3 & 35.6 & $<0.0001$ & 18.8 & 15.1 & 10.5 & $<0.0001$ \\
\hline Low educational level [\%] & 76.3 & 71.4 & 78.2 & $<0.0001$ & 85.5 & 82.8 & 77.6 & $<0.0001$ \\
\hline Not employed [\%] & 28.7 & 25.6 & 19.8 & $<0.0001$ & 55.6 & 51.7 & 47.7 & $<0.0001$ \\
\hline Low social network ${ }^{1}[\%]$ & 53.8 & 48.2 & 45.6 & 0.0004 & 58.1 & 51.7 & 54.4 & $<0.0001$ \\
\hline Living alone [\%] & 20.3 & 16.7 & 17.6 & 0.0457 & 23.9 & 23.2 & 24.7 & 0.5620 \\
\hline High job strain [\%] & 21.8 & 22.6 & 24.2 & 0.3049 & 16.9 & 18.0 & 17.7 & 0.5996 \\
\hline Low self-perceived health [\%] & 20.9 & 16.1 & 15.7 & 0.0016 & 26.9 & 20.5 & 18.9 & $<0.0001$ \\
\hline Depressed mood ${ }^{1}[\%]$ & 34.9 & 35.4 & 33.1 & 0.2693 & 34.8 & 33.5 & 36.7 & 0.2069 \\
\hline High level of somatic compl. ${ }^{1}[\%]$ & 32.9 & 34.4 & 37.1 & 0.0570 & 34.2 & 29.5 & 30.3 & $<0.0018$ \\
\hline
\end{tabular}

Wald $x$-Test.

1 missings $5-<10 \%$.

Crude all-cause mortality rates for six alcohol consumption categories are displayed in Figure 1, indicating a J-shaped curve for men, but not for women. Crude rates of all-cause mortality per 1,000 person-years by three categories of alcohol consumption are presented in Table 2. In men, the crude rate of all-cause mortality was lower in moderate drinkers than in abstainers and heavy drinkers. In women, the crude rate of all-cause mortality was highest in abstainers, whereas no difference between moderate or heavy drinkers could be seen. Compared to women, men with no or moderate alcohol consumption had a twofold higher mortality rate and men with high alcohol consumption a threefold higher mortality rate, respectively.

\section{Cox regression analyses}

The all-cause mortality risks estimated by four Cox proportional hazards models are given in Table 2 showing different results for men and women. For males, moderate alcohol consumers had a significantly reduced risk of mortality from all-causes compared to non-drinkers (HR: 0.68 ; $95 \%$ CI: $0.54-0.85, \mathrm{p}<0.0001$ ) in a 'crude' model (adjusted for age and survey). This association remained stable after adjusting additionally for lifestyle and clinical factors $(H R=0.71,95 \% \mathrm{CI}: 0.56-0.90, \mathrm{p}=0.001)$ or for psychosocial stressors (HR $=0.73,95 \%$ CI: 0.57-0.93, $\mathrm{p}<0.0001$ ). In the 'full' model (adjusted for age, survey, lifestyle, clinical and psychosocial variables), male moderate drinkers still had a significantly reduced mortality risk with a comparable effect (HR: 0.74; 95\% CI: 0.58-0.94, $\mathrm{p}=0.002$ ). In females, moderate drinkers had no significantly reduced risk of all-cause mortality compared to non-drinkers $(\mathrm{HR}=0.80,95 \% \mathrm{CI}: 0.62-1.02, \mathrm{p}=0.189)$. The multivariable adjustment did not affect the relationship between alcohol consumption and mortality in women (see Table 2).

\section{Sensitivity and interaction analyses}

We computed the same models as above with linear variables instead of dichotomized variables as the categorizations of the lifestyle, clinical and psychosocial variables might be too crude. However, we found comparable results. Additional models have been estimated to control for potential differential effects of psychosocial stressors on alcohol consumption by adding interaction terms between alcohol consumption groups and all psychosocial stressors. In men, a significant interaction was found between alcohol consumption and living alone $(\mathrm{p}=0.030)$ : In a stratified analysis, moderate alcohol consumers who lived alone had a reduced risk of all-cause mortality $(\mathrm{HR}=0.48$; 95\% CI $0.28-0.82, \mathrm{p}=0.030)$, whereas subjects not living alone had a HR of 0.85 (95\% CI: 0.641.13). In women, no significant interactions were found between alcohol consumption and psychosocial stressors with respect to all-cause mortality. 
Table 2 Age- and survey- and multivariable-adjusted hazard ratios and $95 \%$ confidence intervals for alcohol consumption in men and women ( $n=11,282$ after exclusion of ill persons at baseline)

\begin{tabular}{|c|c|c|c|c|c|c|c|}
\hline & Number of & Number of & Crude death & Model 1 & Model 2 & Model 3 & Model 4 \\
\hline & & & $\begin{array}{l}\text { rate/1,000 } \\
\text { person-years }\end{array}$ & $\begin{array}{l}\text { Age- and survey- } \\
\text { adjusted }\end{array}$ & $\begin{array}{l}\text { Multivariable- } \\
\text { adjusted }^{2}\end{array}$ & $\begin{array}{l}\text { Multivariable- } \\
\text { adjusted }^{3}\end{array}$ & $\begin{array}{l}\text { Multivariable- } \\
\text { adjusted }^{4}\end{array}$ \\
\hline & & & & HR $(95 \% \mathrm{Cl})^{1}$ & HR $(95 \% \mathrm{Cl})$ & $\mathrm{HR}(95 \% \mathrm{Cl})$ & HR (95\% Cl) \\
\hline \multicolumn{8}{|l|}{ Men $(n=5,540)$} \\
\hline $1: 0 \mathrm{~g} / \mathrm{d}$ & 9,596 & $105 / 850$ & 10.9 & 1.0 & 1.0 & 1.0 & 1.0 \\
\hline $2:>0-39.9 \mathrm{~g} / \mathrm{d}$ & 33,703 & $262 / 2,833$ & 7.8 & $0.68(0.54-0.85)$ & $0.71(0.56-0.90)$ & $0.73(0.57-0.93)$ & $0.74(0.58,0.94)$ \\
\hline $3: \geq 40 \mathrm{~g} / \mathrm{d}$ & 23,406 & $250 / 1,857$ & 10.7 & $0.99(0.79-1.24)$ & $0.94(0.75-1.20)$ & $1.11(0.87-1.42)$ & $1.03(0.80,1.32)$ \\
\hline$p$-value & - & - & - & $<0.0001$ & 0.0010 & $<0.0001$ & 0.0019 \\
\hline \multicolumn{8}{|l|}{ Women $(n=5,742)$} \\
\hline $1: 0 \mathrm{~g} / \mathrm{d}$ & 28,722 & $159 / 2,401$ & 5.5 & 1.0 & 1.0 & 1.0 & 1.0 \\
\hline $2:>0-19.9 \mathrm{~g} / \mathrm{d}$ & 27,790 & $106 / 2,230$ & 3.8 & $0.80(0.62-1.02)$ & $0.87(0.68-1.12)$ & $0.81(0.61-1.07)$ & $0.87(0.66,1.16)$ \\
\hline $3: \geq 20 \mathrm{~g} / \mathrm{d}$ & 14,306 & $55 / 1,111$ & 3.8 & $0.87(0.64-1.18)$ & $0.92(0.67-1.26)$ & $0.86(0.60-1.22)$ & $0.90(0.63,1.29)$ \\
\hline$p$-value & - & - & - & 0.1886 & 0.5541 & 0.3082 & 0.6119 \\
\hline
\end{tabular}

${ }^{1} \mathrm{HR}$ hazard ratio; $\mathrm{Cl}$ confidence interval.

${ }^{2}$ 'Lifestyle/clinical model': Adjusted for age, survey, regular smoking, physical inactivity, obesity, actual hypertension and dyslipidemia.

${ }^{3}$ 'Psychosocial stressors model': Adjusted for age, survey, low educational level, not employed, low social network, living alone, high job strain, low self- perceived health, depressed mood and high level of somatic complaints.

${ }^{4 \prime}$ Full model': Adjusted for age, survey, regular smoking, physical inactivity, obesity, actual hypertension, dyslipidemia, low educational level, not employed, low social network, living alone, high job strain, low self- perceived health, depressed mood and high level of somatic complaints.

\section{Discussion}

\section{Overall}

The present investigation based on a large sample of apparently healthy subjects drawn from the general population confirmed that all-cause mortality is lowest in moderate drinkers, even after controlling for lifestyle and clinical variables as well as for a broad range of psychosocial stressors. Therefore, our data contradict the previously expressed assumption that there is 'probably no free lunch' [39] with drinking alcoholic beverages. To avoid the "sick quitters bias" we excluded subjects reporting disease at baseline as it is possible that these participants quit drinking because of adverse health experiences and therefore could be misclassified. Nonetheless, this exclusion may lead to reduced external validity because the study population does not fully represent the underlying general population anymore.

\section{Lifetime abstainers and ex-drinkers}

The criticism that abstainers per se are a rather heterogeneous group and therefore not an appropriate comparison group is justified since "abstainers" may be lifetime abstainers or ex-drinkers [40]. Because health concerns are frequently related to having given up drinking, it has been argued that a separation of abstainers into lifetime abstainers and ex-drinkers leads to less pronounced or a complete disappearance of beneficial effects [8]. This hypothesis has been attenuated by findings which confirmed a protective association for moderate alcohol consumption and cardiovascular diseases even after separating recent abstainers from lifetime abstainers [41]. A large meta-analysis revealed that ex-drinkers had a higher mortality risk compared to lifetime abstainers. For women, this effect was less pronounced than for men [42]. In our study, after excluding participants reporting severe disease conditions at baseline from all analyses to avoid bias from sick quitters, we still found a J-shaped curve between alcohol consumption and all-cause mortality in men. In women, the J-shaped curve was not approximated, most likely due to a power problem resulting from very small numbers of women in the higher consumption groups. However, there might be several differences between lifetime abstainers and ex-drinkers which we were not able to account for such as previous problematic drinking patterns among ex-drinkers. Similar risks for negative health conditions were shown for former heavy drinkers compared to current heavy drinkers [43].

\section{Lifestyle factors}

The present investigation confirmed earlier findings that male moderate drinkers have a positive lifestyle behaviour that favours their survival over non-drinkers (e.g. as physical activity and non-smoking behaviour) [44-49]. The benefits of moderate drinking relative to abstinence were present primarily within the context of an otherwise healthy behavioural profile [50].

\section{Psychosocial stressors}

The hypothesis of the modification of the risk curve between alcohol and all-cause mortality by social isolation $[51,52]$ has been empirically tested, but a direct protective effect of social integration has not been confirmed 
$[53,54]$. In our data, we found that the effect of alcohol consumption on all-cause mortality was modified by living alone or not alone in men: Whereas men who lived alone had a significantly reduced mortality risk compared to men reporting no alcohol consumption. No significant differences in mortality risk were found for the three alcohol consumption groups in men living not alone. Therefore, alcohol consumption had no effect on mortality in men living not alone. One explanation for this finding might be that men who did not live alone already had a reduced mortality risk compared to men living alone and therefore, there was no space for a significant decreased mortality risk by moderate alcohol consumption (however, a tendency toward risk reduction was found with HR 0.85).

There is evidence from several cross-sectional studies showing that non-drinkers as well as heavy drinkers experience higher levels of depression, psychological distress, anxiety and lower levels of subjective health than moderate drinkers [20-26,55]. In our study, there is no clear evidence of a generally lower level of psychosocial stressors in moderate drinkers in comparison to nondrinkers and heavy drinkers. Moderate drinkers reported somatic complaints less frequently, however, for depressed mood, no differences between the three consumption categories were found. The lack of significant differences in depressed mood and other measures of psychological distress in our study as opposed to other studies remains unclear; the use of different instruments might contribute to these inconsistencies. However, the present investigation did not provide compelling evidence that the J- or U-shaped relationship between alcohol and all-cause mortality could be explained by confounding from psychosocial stressors.

\section{Potential pathophysiological mechanism}

Several pathophysiological potential mechanisms for the protective effect of moderate alcohol intake have been suggested [7]. The J-shape of mortality risk has been attributed to a combination of beneficial and harmful effects of ethanol itself. Among them are lower levels of inflammatory markers, improved flow-mediated vasodilation and favourable effects on serum lipid levels for subjects having a low to moderate alcohol intake compared to abstainers [56].

In addition to overall level of alcohol consumption, specific drinking patterns, especially heavy or so-called binge drinking, has also been shown to affect mortality; meta-analyses revealed lower cardiovascular risks as a result of regular daily intake of a low to moderate dose of alcohol, and a higher risk from infrequent binge drinking [57-59]. Overall, the causes of the advantageous health status of moderate drinkers must be regarded as multiple and should be further investigated.

\section{Strengths and limitations}

The strengths of the present study are the prospective design, the large sample size based on a random sample drawn from the general population and the availability of a large set of lifestyle and clinical factors as well as numerous external and internal psychosocial stressors. Additionally, all factors were scrutinized by standardized and quality-controlled assessments. The large sample size allows a broad controlling for potential confounders. Excluding subjects already diseased at baseline from the analyses avoided misattribution of psychosocial variables to abstinence, as diseases could both lead to abstention and development of mood disorders or impaired social conditions.

The MONICA/KORA Augsburg Cohort Study has several limitations that need to be considered. Because the study was limited to men and women of German nationality, caution should be used in generalizing these results to people of other ethnicities. A general limitation of studies on alcohol is that self-reported alcohol intake is particularly susceptible to underreporting [60], not only due to selection and recall bias but also due to a tendency to give socially desirable answers. However, comparing the proportion of persons with elevated serum gamma-glutamyltransferase in the three alcohol consumption groups in S1 did not indicate a misattribution of drinkers in the abstinent group (data not shown). In addition, we couldn't perform a multiple measurement of alcohol intake but only a single week and only a single day/weekend measurement. Therefore, only volume could have been analysed and not specific drinking patterns such as heavy drinking episodes. Furthermore, a major shortcoming is the fact that no differentiation between lifetime abstainers and current abstainers could be made. Another limitation that needs to be addressed is that depressive symptoms were measured using a symptom rating scale which is among the less rigorous options to assess depressive mood, although a recent re-examination of its validity and reliability is promising [37]. The results do not pertain to major depression as defined in international classification systems. Additionally, depressive symptoms were measured at one time point, so that transient states of depression could not be distinguished from persistent states.

\section{Conclusion}

The present study analysed the impact of psychosocial stressors on the association of alcohol and all-cause mortality after excluding potential 'sick quitters'. In men, moderate drinkers were at significantly lower all-cause mortality risk than non-drinkers or heavy drinkers. This pattern persisted after adjustment for lifestyle risk factors and clinical as well as for external and internal psychosocial stressors. In women, no protective effect of 
moderate drinking was shown. The observed protective effect of moderate drinking could not be attributed to misclassification or confounding by psychosocial stressors in a large cohort of men and women drawn from the general population in Southern Germany.

\section{Competing interests}

The authors declare that they have no competing interests.

\section{Authors' contributions}

ER prepared the data, performed statistical analyses of the data, contributed to the interpretation of the findings and drafted the paper. JB performed statistical analyses of the data, contributed to the interpretation of the results and writing of the manuscript. CM and $A D$ contributed to the interpretation of the findings. KHL had the idea of the study, supervised the study and contributed to the interpretation of the findings. All authors read and approved the final draft of the paper.

\section{Acknowledgements}

The MONICA/KORA Augsburg Cohort Study is financed by the Helmholtz Zentrum München, German Research Center for Environmental Health (formerly GSF-National Research Center for Environment and Health). We are grateful to the MONICA/KORA Augsburg teams, which have conducted the studies and identified the clinical outcomes of the survey participants. We thank Ulrich Keil, MD, who initiated the MONICA Study Augsburg as principal investigator. Finally, we thank Andrea Schneider for data handling and quality control and Alexander von Eisenhart Rothe, MPH, for language editing.

\section{Author details}

'Institute of Epidemiology II, Helmholtz Zentrum München, German Research Center for Environmental Health, Ingolstädter Landstr. 1, 85764 Neuherberg, Germany. ${ }^{2}$ MONICA/KORA Myocardial Infarction Registry, Central Hospital Augsburg, Stenglinstr 2, 86156 Augsburg, Germany. ${ }^{3}$ Institute of Epidemiology I, Helmholtz Zentrum München, German Research Center for Environmental Health, Ingolstädter Landstr. 1, 85764 Neuherberg, Germany. ${ }^{4}$ Department of Psychosomatic Medicine and Psychotherapy, Klinikum rechts der Isar, Technische Universität München, Langerstr. 3, 81675 München, Germany.

Received: 22 May 2013 Accepted: 19 March 2014

Published: 4 April 2014

\section{References}

1. Keil U, Chambless LE, Doring A, Filipiak B, Stieber J: The relation of alcohol intake to coronary heart disease and all-cause mortality in a beer-drinking population. Epidemiology 1997, 8:150-156.

2. Rehm J, Baliunas D, Borges GL, Graham K, Irving H, Kehoe T, Parry CD, Patra J, Popova S, Poznyak V, Roerecke M, Room R, Samokhvalov AV, Taylor B: The relation between different dimensions of alcohol consumption and burden of disease: an overview. Addiction 2010, 105:817-843.

3. Ronksley PE, Brien SE, Turner BJ, Mukamal KJ, Ghali WA: Association of alcohol consumption with selected cardiovascular disease outcomes: a systematic review and meta-analysis. BMJ 2011, 342:d671

4. Di Castelnuovo A, Costanzo S, Bagnardi V, Donati MB, lacoviello L, de Gaetano G: Alcohol dosing and total mortality in men and women: an updated meta-analysis of 34 prospective studies. Arch Intern Med 2006, 166:2437-2445.

5. Rehm J, Gmel G, Sempos CT, Trevisan M: Alcohol-related morbidity and mortality. Alcohol Res Health 2003, 27:39-51.

6. Gronbaek M: The positive and negative health effects of alcohol and the public health implications. J Intern Med 2009, 265:407-420.

7. Kloner RA, Rezkalla SH: To drink or not to drink? That is the question. Circulation 2007, 116:1306-1317.

8. Shaper AG, Wannamethee G, Walker M: Alcohol and mortality in British men: explaining the U-shaped curve. Lancet 1988, 2:1267-1273.

9. Strandberg TE, Strandberg AY, Salomaa W, Pitkala K, Tilvis RS, Miettinen TA: Alcoholic beverage preference, 29-year mortality, and quality of life in men in old age. J Gerontol A Biol Sci Med Sci 2007, 62:213-218.

10. Andreasson S: Alcohol and J-shaped curves. Alcohol Clin Exp Res 1998, 22:359S-364S
11. Pulska T, Pahkala K, Laippala P, Kivelä SL: Follow up study of longstanding depression as predictor of mortality in elderly people living in the community. BMJ 1999, 318:432-433.

12. Zheng D, Macera CA, Croft JB, Giles WH, Davis D, Scott WK: Major depression and all-cause mortality among white adults in the United States. Ann Epidemiol 1997, 7:213-218.

13. Cuijpers P, Smit F: Excess mortality in depression: a meta-analysis of community studies. J Affect Disord 2002, 72:227-236.

14. Kivimaki M, Leino-Arjas $P$, Luukkonen R, Riihimaki H, Vahtera J, Kirjonen J: Work stress and risk of cardiovascular mortality: prospective cohort study of industrial employees. BMJ 2002, 325:857.

15. Kivimaki M, Virtanen $M$, Elovainio M, Kouvonen A, Vaananen A, Vahtera J: Work stress in the etiology of coronary heart disease - a meta-analysis. Scand J Work Environ Health 2006, 32:431-442.

16. Huppert FA, Whittington JE: Symptoms of psychological distress predict 7-year mortality. Psychol Med 1995, 25:1073-1086.

17. Kaplan GA, Camacho T: Perceived health and mortality: a nine-year follow-up of the human population laboratory cohort. Am J Epidemiol 1983, 117:292-304

18. Eaker ED, Sullivan LM, Kelly-Hayes M, D'Agostino RB Sr, Benjamin EJ: Tension and anxiety and the prediction of the 10-year incidence of coronary heart disease, atrial fibrillation, and total mortality: the Framingham Offspring Study. Psychosom Med 2005, 67:692-696.

19. Allgulander C, Lavori PW: Excess mortality among 3302 patients with 'pure' anxiety neurosis. Arch Gen Psychiatry 1991, 48:599-602.

20. Naimi TS, Brown DW, Brewer RD, Giles WH, Mensah G, Serdula MK, Mokdad AH, Hungerford DW, Lando J, Naimi S, Stroup DF: Cardiovascular risk factors and confounders among nondrinking and moderate-drinking U.S. adults. Am J Prev Med 2005, 28:369-373.

21. Lipton Rl: The effect of moderate alcohol use on the relationship between stress and depression. Am J Public Health 1994, 84:1913-1917.

22. Lyons RA, Lo SV, Monaghan S, Littlepage BN: Mortality and alcohol consumption. Moderate drinking also improves health. BMJ 1995, 310:326.

23. Power C, Rodgers B, Hope S: U-shaped relation for alcohol consumption and health in early adulthood and implications for mortality. Lancet 1998, 352:877.

24. Rodgers B, Korten AE, Jorm AF, Jacomb PA, Christensen H, Henderson AS: Non-linear relationships in associations of depression and anxiety with alcohol use. Psychol Med 2000, 30:421-432.

25. Leifman $\mathrm{H}$, Kuhlhorn $\mathrm{E}$, Allebeck $\mathrm{P}$, Andreasson $\mathrm{S}$, Romelsjo A: Abstinence in late adolescence - antecedents to and covariates of a sober lifestyle and its consequences. Soc Sci Med 1995, 41:113-121.

26. Peele S, Brodsky A: Exploring psychological benefits associated with moderate alcohol use: a necessary corrective to assessments of drinking outcomes? Drug Alcohol Depend 2000, 60:221-247.

27. Colder CR: Life stress, physiological and subjective indexes of negative emotionality, and coping reasons for drinking: Is there evidence for a self-medication model of alcohol use? Psychol Addict Behav 2001, 15:237-245.

28. WHO MONICA Project Principal Investigators (prepared by H. Tunstall-Pedoe): Myocardial Infarction and coronary deaths in the World Health Organization MONICA Project. Registration procedures, event rates, and case fatality rates in 38 populations from 21 countries in four continents. Circulation 1994, 90:538-612.

29. Holle R, Happich M, Lowel H, Wichmann HE, MONICA/KORA Study Group: KORA - a research platform for population based health research. Gesundheitswesen 2005, 67:S19-S25.

30. WHO MONICA Project Principal Investigators: MONICA Psychosocial Optional Study. Suggested Measurement Instruments. Copenhagen: WHO Regional Office for Europe; 1989

31. Loewel H, Lewis M, Hoermann A, Keil U: Case finding, data quality aspects and comparability of myocardial infarction registers: results of a south German register study. J Clin Epidemiol 1991, 44:249-260.

32. Wellmann J, Heidrich J, Berger K, Doring A, Heuschmann PU, Keil U: Changes in alcohol intake and risk of coronary heart disease and all-cause mortality in the MONICA/KORA-Augsburg cohort 1987-97. Eur J Cardiovasc Prev Rehabil 2004, 11:48-55.

33. Seeman TE, Kaplan GA, Knudsen L, Cohen R, Guralnik J: Social network ties and mortality among the elderly in the Alameda County Study. Am J Epidemiol 1987, 126:714-723.

34. Karasek A: Job demands, job decision latitude, and mental strain: implications for job redesign. Administration SC Q 1979, 24:285-307. 
35. Heidrich J, Liese AD, Lowel H, Keil U: Self-rated health and its relation to all-cause and cardiovascular mortality in southern Germany. Results from the MONICA Augsburg cohort study 1984-1995. Ann Epidemiol 2002, 12:338-345

36. Zerssen D v: Die Beschwerden-Liste. Klinische Selbstbeurteilungsfragebögen aus dem Münchner Psychiatrischen Informationssystem. In Psychis Manuale, Allgemeiner Teil. Weinheim: Beltz; 1976.

37. Ladwig KH, Marten-Mittag B, Baumert J, Doering A, Loewel H: Case-finding for vital exhaustion and depressive mood in the general population: reliability and validity of a symptom driven diagnostic scale. Results from the MONICA-KORA Augsburg Study. Ann Epidemiol 2004, 14:332-338.

38. Breslow NE, Day NE: Statistical Methods in Cancer Research. Volume II The Design and Analysis of Cohort Studies. IARC Sci Pub/ Nr. 82. Lyon, France: IARC; 1987.

39. Jackson R, Broad J, Connor J, Wells S: Alcohol and ischaemic heart disease: probably no free lunch. Lancet 2005, 366:1911-1912

40. Huth C, Siegert N, Meisinger C, Konig J, Kaab S, Wichmann HE, Doring A: Individuals with very low alcohol consumption: a heterogeneous group. I Stud Alcohol Drugs 2007, 68:6-10.

41. Thompson PL: J-curve revisited: cardiovascular benefits of moderate alcohol use cannot be dismissed. Med J Aust 2013, 198:419-422.

42. Gmel G, Gutjahr E, Rehm J: How stable is the risk curve between alcohol and all-cause mortality and what factors influence the shape? A precision-weighted hierarchical meta-analysis. Eur J Epidemiol 2003, 18:631-642.

43. Lown ES, Greenfield TK, Rogers JD: Health effects from drinking: type, severity, and associated drinking patterns based on qualitative and qantitative questions in a methodological survey. Subst Use Misuse 2007, 42:793-810.

44. Camargo CA Jr, Hennekens CH, Gaziano JM, Glynn RJ, Manson JE, Stampfer MJ: Prospective study of moderate alcohol consumption and mortality in US male physicians. Arch Intern Med 1997, 157:79-85.

45. Gronbaek M, Deis A, Sorensen TI, Becker U, Schnohr P, Jensen G: Mortality associated with moderate intakes of wine, beer, or spirits. BMJ 1995 310:1165-1169.

46. Solomon CG, Hu FB, Stampfer MJ, Colditz GA, Speizer FE, Rimm EB, Willett WC, Manson JE: Moderate alcohol consumption and risk of coronary heart disease among women with type 2 diabetes mellitus. Circulation 2000, 102:494-499.

47. Kearney J, Giovannucci E, Rimm EB, Stampfer MJ, Colditz GA, Ascherio A Bleday R, Willett WC: Diet, alcohol, and smoking and the occurrence of hyperplastic polyps of the colon and rectum (United States). Cancer Causes Control 1995, 6:45-56.

48. Barefoot JC, Gronbaek M, Feaganes JR, McPherson RS, Williams RB, Siegler IC: Alcoholic beverage preference, diet, and health habits in the UNC Alumni Heart Study. Am J Clin Nutr 2002, 76:466-472.

49. Tjonneland A, Gronbaek M, Stripp C, Overvad K: Wine intake and diet in a random sample of 48763 Danish men and women. Am J Clin Nutr 1999, 69:49-54.

50. Shaw BA, Agahi N: A prospective cohort study of health behavior profiles after age 50 and mortality risk. BMC Public Health 2012, 12:803.

51. Skog OJ: The J-curve, causality and public health. Addiction 1995, 90:490-492.

52. Skog OJ: Public health consequences of the J-curve hypothesis of alcohol problems. Addiction 1996, 91:325-337.

53. Murray RP, Rehm J, Shaten J, Connett JE: Does social integration confound the relation between alcohol consumption and mortality in the Multiple Risk Factor Intervention Trial (MRFIT)? J Stud Alcohol 1999, 60:740-745.

54. Greenfield TK, Rehm J, Rogers JD: Effects of depression and social integration on the relationship between alcohol consumption and all-cause mortality. Addiction 2002, 97:29-38.

55. Caldwell TM, Rodgers B, Jorm AF, Christensen $H$, Jacomb PA, Korten AE: Patterns of association between alcohol consumption and symptoms of depression and anxiety in young adults. Addiction 2002, 97:583-594.

56. Brien SE, Ronksley PE, Turner BJ, Mukamal KJ, Ghali WA: Effect of alcohol consumption on biological markers associated with risk of coronary heart disease: systematic review and meta-analysis of interventional studies. BMJ 2011, 342:d636.

57. Murray RP, Connett JE, Tyas SL, Bond R, Ekuma O, Silversides CK, Barnes GE: Alcohol volume, drinking pattern, and cardiovascular disease morbidity and mortality: is there a U-shaped function? Am J Epidemiol 2002, 155:242-248.

58. Bagnardi V, Zatonski W, Scotti L, La Vecchia C, Corrao G: Does drinking pattern modify the effect of alcohol on the risk of coronary heart disease? Evidence from a meta-analysis. J Epidemiol Community Health 2008, 62:615-619.

59. Roerecke $M$, Rehm J: Irregular heavy drinking occasions and risk of ischemic heart disease: a systematic review and meta-analysis. Am J Epidemiol 2010, 171:633-644.

60. Pernanen K: Validity of survey data on alcohol use. In Research advances in alcohol and drug problems. Edited by Gibbins R, Israel Y, Kalant H, Sellers EM. New York, NY: John Wiley and Sons, Inc; 1974:355-374.

doi:10.1186/1471-2458-14-312

Cite this article as: Ruf et al:: Are psychosocial stressors associated with the relationship of alcohol consumption and all-cause mortality? BMC Public Health 2014 14:312.

\section{Submit your next manuscript to BioMed Central and take full advantage of:}

- Convenient online submission

- Thorough peer review

- No space constraints or color figure charges

- Immediate publication on acceptance

- Inclusion in PubMed, CAS, Scopus and Google Scholar

- Research which is freely available for redistribution 